\title{
Theoretical evaluation on potential cytotoxicity of graphene quantum dots
}

Lijun Liang ${ }^{1, *}$, Zhe Kong ${ }^{2}$, Zhengzhong Kang ${ }^{3,}{ }^{4}$, Hongbo Wang ${ }^{5}$, Li Zhang ${ }^{6}$, Jia-Wei Shen ${ }^{7, *}$

${ }^{1}$ College of Life Information Science and Instrument Engineering, Hangzhou Dianzi University, No. 1, 2nd Street, Jianggan District, Hangzhou, 310018, People's Republic of China

${ }^{2}$ College of Materials and Environmental Engineering, Hangzhou Dianzi University, Hangzhou, No. 1, 2nd Street, Jianggan District, Hangzhou, 310018, People's Republic of China

${ }^{3}$ Department of Chemistry, Zhejiang University, Zheda Road 38, Hangzhou, 310028, People's Republic of China

${ }^{4}$ Division of Theoretical Chemistry and Biology, School of Biotechnology, KTH Royal Institute of Technology, SE-10691 Stockholm, Sweden

${ }^{5}$ College of Automation, Hangzhou Dianzi University, No. 1, 2nd Street, Jianggan District, Hangzhou 310018, People's Republic of China

${ }^{6}$ Department of Chemistry, Zhejiang Sci-Tech University, No. 2, 2nd Street, Jianggan District, Hangzhou, 310012, People's Republic of China

${ }^{7}$ School of Medicine, Hangzhou Normal University, Xuelin $\quad$ street $\quad 16, \quad$ Jianggan District, Hangzhou 310016, People's Republic of China

Email address:

michael.lijunl@gmail.com (L. Liang)

shen.jiawei@hotmail.com (J.-W. Shen) 


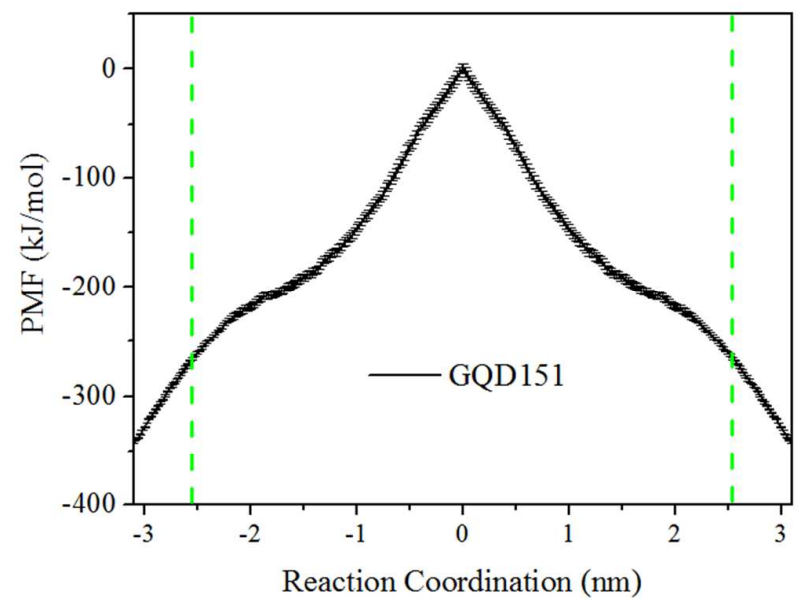

Figure S1. PMF of GQD151 translocating through POPC membrane.

As we discussed in the manuscript, the free energy barrier increases with the increase of GQDs size, and for GQD151 translocating through POPC membrane, the energy barrier is around $350 \mathrm{~kJ} / \mathrm{mol}$. This indicates that the translocating of GQD151 across POPC membrane is extremely difficult and highly prohibited. Meanwhile, due to highly unstable structure of GQD151 staying in the middle of POPC membrane, the calculation of PMF of GQD151 is not as accurate as PMF of GQD with smaller size. Therefore, the PMF of GQD with larger size than GQD151 is hard to accurately calculate and is not reliable. However, one could infer that it is more difficult for QGDs with larger size translocating through POPC membrane on the basis of Figure 4 in the manuscript and our discussion. 

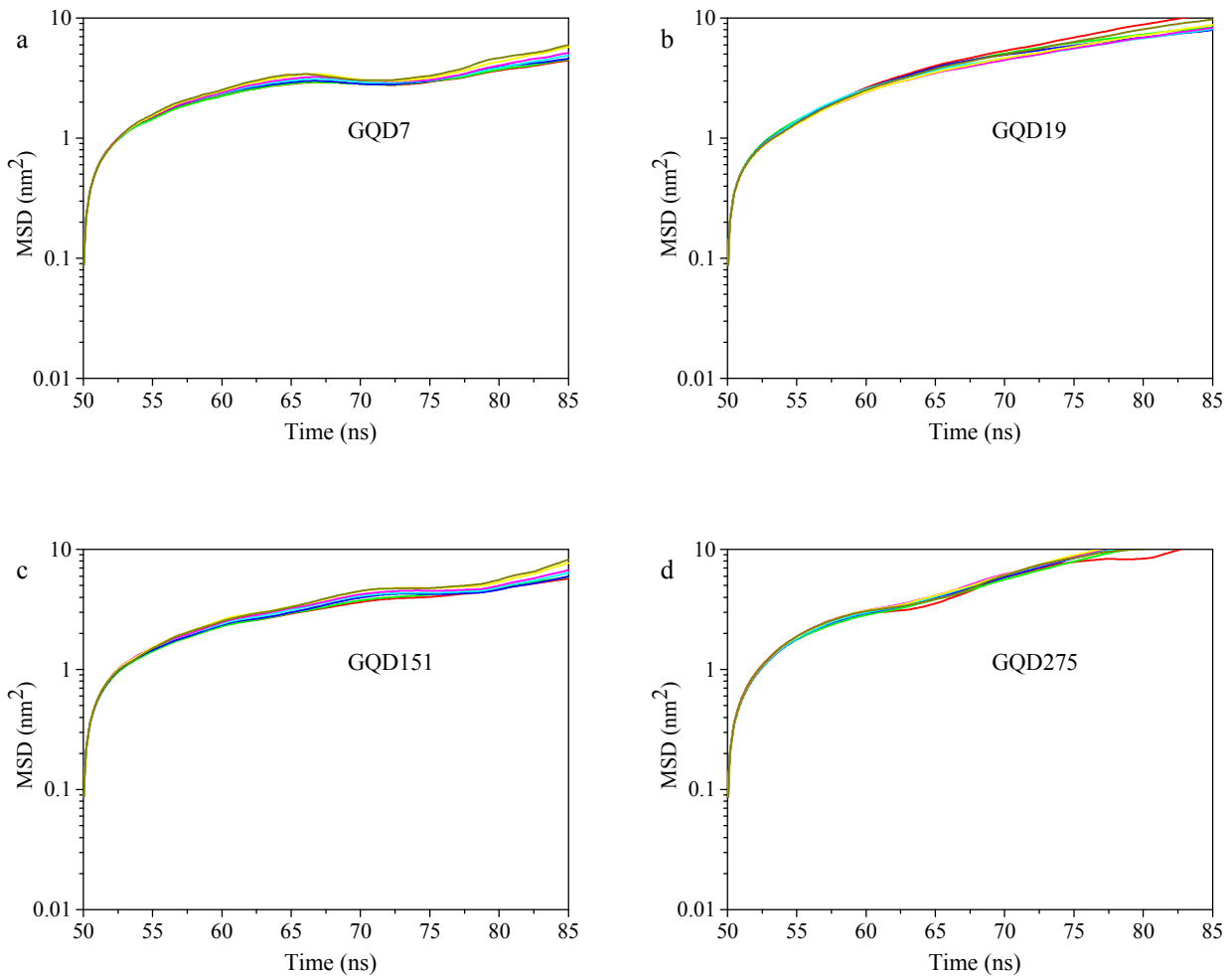

Figure S2. The MSD (mean squared displacement) of lipid membrane in $x-y$ plane as a function of simulation time in different systems in seven parallel simulations: GQD7 (black line), GQD19 (red line), GQD151 (pink line) and GQD275 (blue line). 

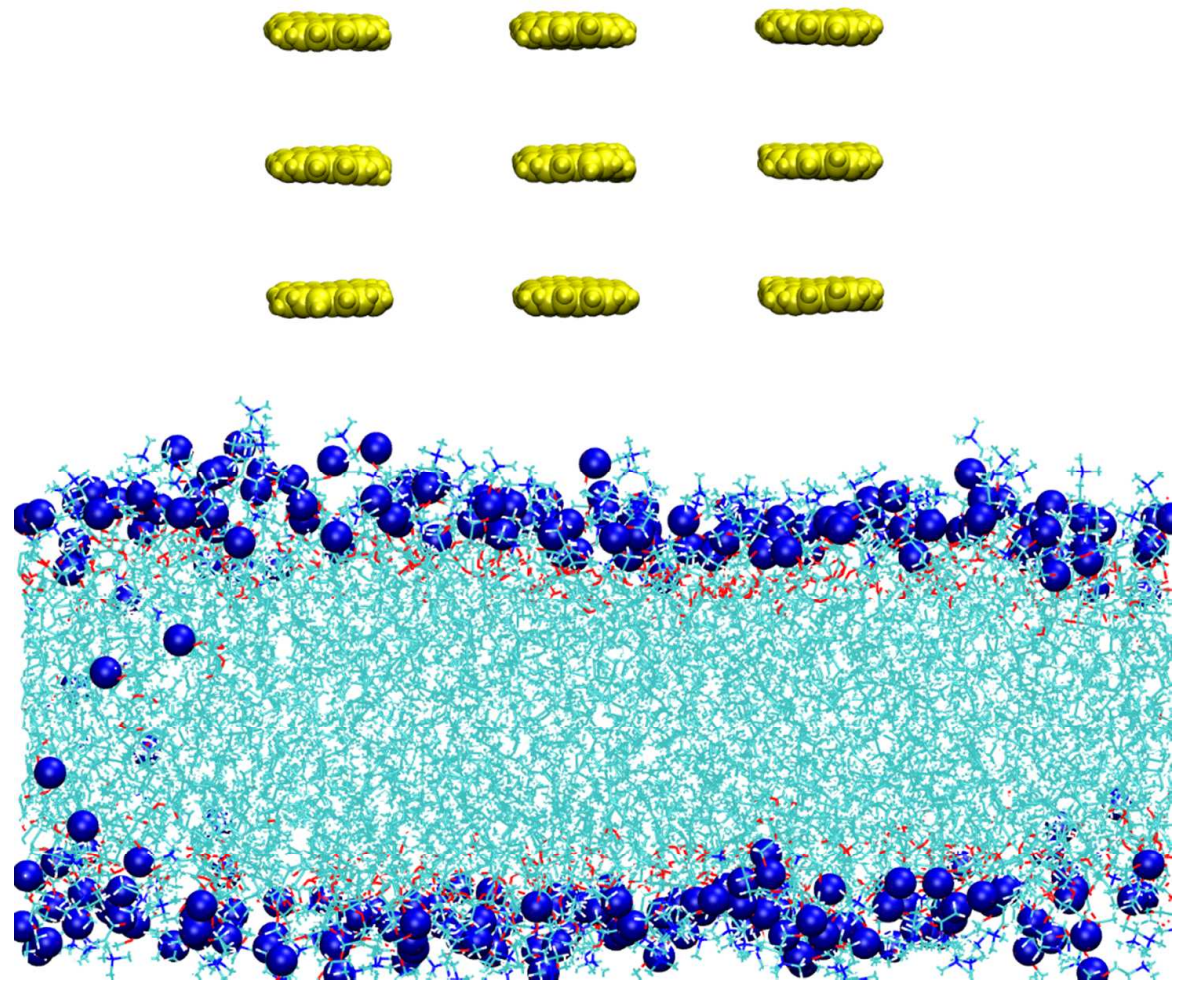

Figure S3. The initial structure of multiple GQDs on top of POPC membrane. GQDs were represented by yellow vdW model, POPC membrane were represented by line model, and $\mathrm{P}$ atoms in membrane were represented by blue vdW model. Water molecules were not shown for clarity.
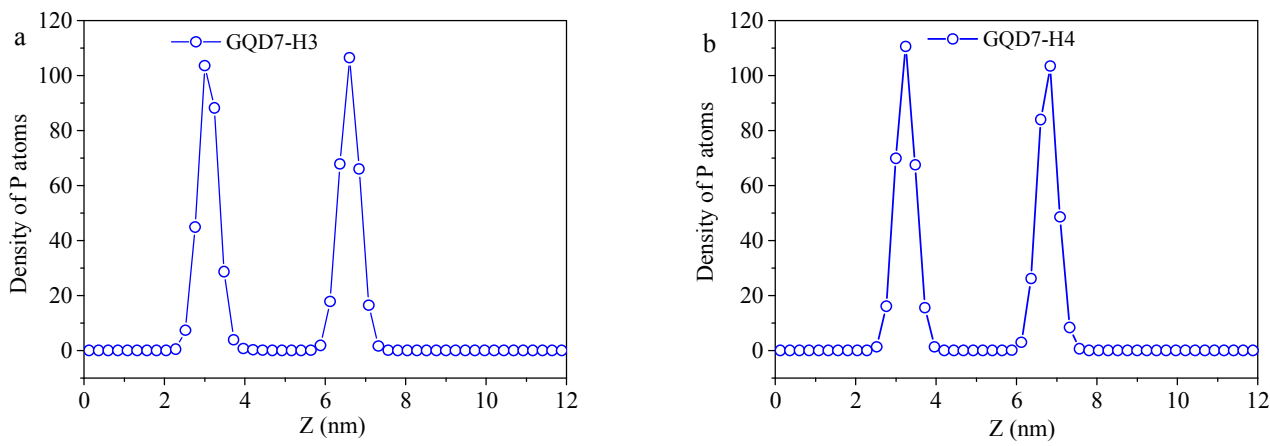

Figure S4. The density of $\mathrm{P}$ atoms in $z$ direction. The distance between two highest points was considered as the thickness of lipid bilayer. (a) GQD7-H3; (b) GQD7-H4. 


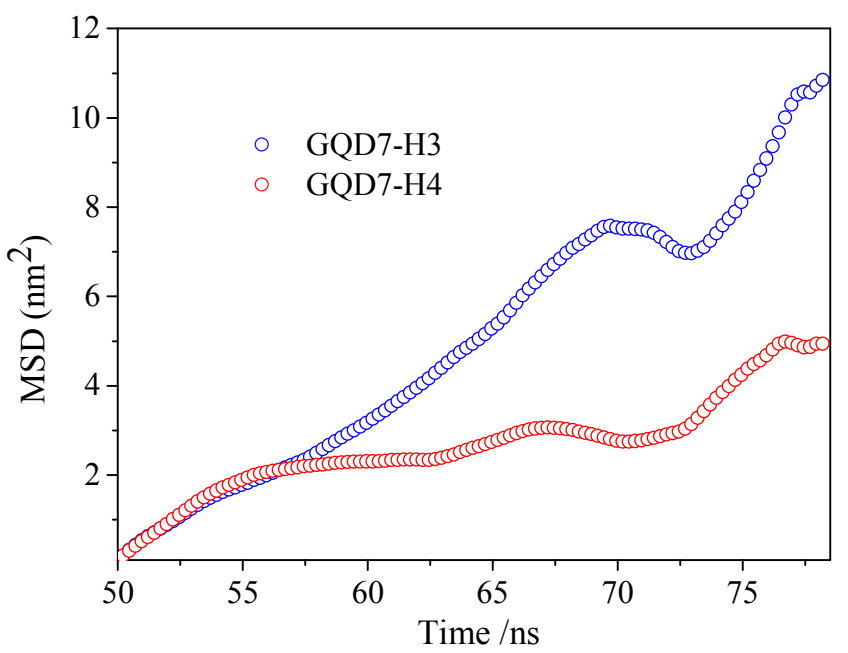

Figure S5. The MSD (mean squared displacement) of lipid membrane in $x-y$ plane as a function of simulation time in different systems: GQD7-H3 (blue circle), and GQD7-H4 (red circle). 